\title{
When are supercomputers worth the bother?
}

\author{
JEFFREY B. MULLIGAN \\ NASA-Ames Research Center, Moffett Field, Califormia
}

\begin{abstract}
The availability of the UNIX operating system on large supercomputers has greatly simplified the importation of research software from workstations and minicomputers. Supercomputers generally offer much greater memory and storage than do smaller machines, which may make their use mandatory for some large applications. Realization of the full performance offered by vectorizing supercomputers such as the Cray C90 sometimes requires modification of existing code. The use of high-level interpreters for the development of applications is recommended, to minimize the amount of compiled code that must be optimized.
\end{abstract}

For those with access, supercomputers offer high computing power and large capacity. But such attributes are sometimes offset by the high cost of having to adapt software. In this paper we examine some of the tradeoffs in supercomputer use.

In recent years, the power of desktop computers has increased dramatically; it is to be hoped that future increases will continue at a similar rate. The concept of the supercomputer is thus somewhat slippery, since today's workstation would have been considered a supercomputer 10 years ago, and today's supercomputer may fit on a single chip 10 years hence. Here the term supercomputer will refer to a machine so expensive that it is not an option for an individual, or even a group of researchers, to purchase one for exclusive use. Such machines are found only in computing centers that serve many users. As one would hope, their high cost brings with it unique features that are imperative for certain applications. For other applications, these machines may offer improved performance on a job that could be done on a smaller machine. And for other applications, the performance gain may be small-if any work at all is required to make the program run on the new machine ("porting"), it may not be worth the trouble.

Here I will focus on the Cray C90 at Ames Research Center. This machine's primary feature is vectorization hardware, whereby a series of numerical operations can be executed in parallel. To support large physical simulations, a correspondingly large amount of memory and disk storage is provided (see Table 1). In general, code written for this system looks similar to that for smaller machines and will run on them; the compiler automatically determines when the machine should vectorize. To optimize performance, however, the programmer will want to read the report of the optimizations done by the compiler in order to ensure that all desired vectorizations are indeed occurring. Certain codes may have to be rewritten to make this happen.

Correspondence concerning this paper should be addressed to J. B. Mulligan, NASA-Ames Research Center, MS 262-2, Moffett Field, CA 94035-1000 (e-mail: jbm@vision.arc.nasa.gov).
The Cray C90 can be contrasted with another type of supercomputer, the massively parallel machine exemplified by the Connection Machine, which consists of thousands of relatively simple processors. The architecture of this machine is inherently unsuited to traditional linear programming languages, which has necessitated the introduction of language extensions to exploit the special features of such machines. The resulting programs can no longer be run "as is" on a traditional machine, although emulators are available which allow development and debugging to be done on workstations.

Because supercomputer time is usually at a premium, interoperability (the ability to test programs on personal systems) is important to consider. Cray's adoption of UNIX as the basis of their UNICOS operating system was a major step in this direction, providing an environment similar to that of most workstations. An important aspect of a common operating system is that users do not have to learn and remember two sets of commands for file manipulation and other housekeeping functions. This also makes porting applications from workstations up to the Cray simpler in most cases than porting down to DOS or Macintosh environments. Application programs that are easily ported allow development and debugging of a small-scale version to be done on a local workstation, followed by a fullscale run on the supercomputer.

In keeping with this philosophy, and to minimize the amount of code that must be ported, it is often useful to concentrate coding efforts on the development of fairly general-purpose interpretive programs. Current commercially available examples include systems such as Matlab and Mathematica, but the idea can be extended to locally developed codes. The fundamental idea is to have a single copy of the often used routines and use a text interpreter to control the sequencing. Specific applications are then coded in the scripting language instead of the lowlevel programming language. In addition to producing a cleaner, more readable program that is easy to understand and modify, these scripted applications are transparently portable once the interpreter itself has been ported. This philosophy has driven the development here at Ames of an interactive image-processing system known 
Table 1

Comparison of Example Workstations and Cray Supercomputer, With Run Times on a Simple Problem

\begin{tabular}{lrrrr}
\hline & & \multicolumn{3}{c}{ Compute Throughput } \\
\cline { 3 - 5 } Machine & RAM & $\begin{array}{c}\text { Elapsed } \\
\text { Time }\end{array}$ & $\begin{array}{c}\text { CPU } \\
\text { Utilization }\end{array}$ & $\begin{array}{c}\text { Throughput } \\
\text { (Mflops) }\end{array}$ \\
\hline $\begin{array}{l}\text { SUN Sparc2 } \\
\text { SGI Indigo2 }\end{array}$ & 64 & $2: 18$ & $93 \%$ & 0.7 \\
$\begin{array}{c}\text { Cray C90 } \\
\text { (unvectorized) }\end{array}$ & 1,600 & $0: 24$ & $88 \%$ & 1.6 \\
$\begin{array}{c}\text { Cray C90 } \\
\text { (vectorized) }\end{array}$ & 1,600 & $0: 03$ & $37 \%$ & 5 \\
\hline
\end{tabular}

Note-RAM is actual memory in megabytes installed in the test systems, which is not necessarily the maximum capacity of a fully populated system. CPU utilization is the percentage of the elapsed time during which the CPU was active on the test job; these numbers fall below $100 \%$ when the CPU has to wait for input/output activity (e.g., disk access) to complete, or because the CPU is shared with other users.

as QuIP (QUick Image Processing), which consists of a number of modules coded in $\mathrm{C}$, including a text interpreter that controls the other functions.

Although text interpretation imposes additional overhead (compared to the use of a compiled program), this is often insignificant when the interpreted directives refer to the large, computationally intensive operations, that are most likely to be run on supercomputers. This is particularly true of image processing, where a simple statement requesting that two images be added together may result in a quarter of a million floating point operations.

The QuIP interpreter was used to produce the performance comparison given in Table 1. A simple script was written that created two $1 \mathrm{~K} \times 1 \mathrm{~K}$ images, initialized them, and computed the pixelwise product 100 times. The time to process 1 million pixels (one image's worth) is obtained by dividing the running time by 100 , and the reciprocal of this number is the throughput in megaflops (floating point operations per second). (The numbers thus obtained are lower than the various manufacturers' specifications, because the inner loops of the QuIP routines are written to handle objects with noncontiguous data.)

As Table 1 shows, while the unvectorized performance of the Cray C90 is nothing to sneeze at, it is in the same ballpark as that of the workstations (and higher end workstations on the market today can duplicate this performance). The vectorized performance, on the other hand, is almost two orders of magnitude better than the workstations' performance, and for many jobs this may represent a worthwhile speedup. Unfortunately, this performance was not obtained when the current version of the software was recompiled on the Cray, and it may be instructive to see why not. The C code for the inner loop of the routine that does the multiplication was something like the following fragment:

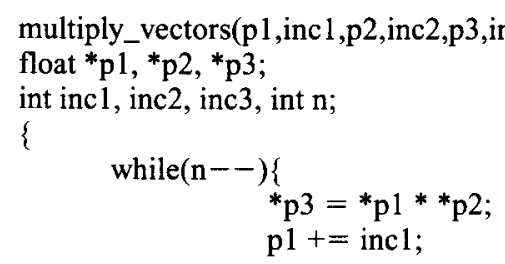

float *p1, *p2, *p3;

int inc1, inc2, inc3, int $\mathrm{n}$;

$$
\begin{aligned}
\text { while }(\mathrm{n}--) & \left\{\begin{aligned}
{ }^{\mathrm{p}} 3 & ={ }^{*} \mathrm{p} 1{ }^{*} \mathrm{*} 2 \\
\mathrm{p} 1+ & =\mathrm{inc} 1 ;
\end{aligned}\right.
\end{aligned}
$$

$\mathrm{p} 2+=$ inc2;

$$
\text { p3 += inc3; }
$$

\section{\}}

While this subroutine may be somewhat obscure to readers not familiar with pointers and $C$, the function is fairly simple: the addresses of three arrays of data are passed in the pointer variables $\mathrm{p} 1, \mathrm{p} 2$, and $\mathrm{p} 3$. The arrays are scanned, with the product of the entries pointed to by $\mathrm{p} 1$ and $\mathrm{p} 2$ being deposited in the location pointed to by $\mathrm{p} 3$. After each multiplication, the pointers are incremented by the arguments inc 1 , inc 2 , and inc3.

Why does this subroutine not vectorize as written? The key to successful vectorization is that the values of the variables in a given iteration must not depend on values computed in previous iterations. In the preceding example, the value of pointer $\mathrm{pl}$ is incremented by incl; that is, the new value is the value from the last iteration, plus incl. In this case, because the increment is not changed, it is possible to determine ahead of time what the values of the pointers will be in every iteration, but unfortunately the compiler is not smart enough to recognize this on its own! The following code fragment does the same thing, but is vectorized by the compiler:

$$
\begin{aligned}
& \text { multiply_vectors(p1,inc1,p2,inc2,p3,inc3,n) } \\
& \text { float }{ }^{*} \mathrm{p} 1,{ }^{*} \mathrm{p} 2,{ }^{*} \mathrm{p} 3 \text {; } \\
& \text { int inc1, inc2, inc3, int } \mathrm{n} \text {; } \\
& \{ \\
& \text { int } \mathrm{i}, \mathrm{i} 1=0, \mathrm{i} 2=0, \mathrm{i} 3=0 \text {; } \\
& \text { for }(i=0 ; i<n ; i++)\{ \\
& \mathrm{p} 3[\mathrm{i} 3]=\mathrm{p} 1[\mathrm{i} 1] * \mathrm{p} 2[\mathrm{i} 2] \text {; } \\
& \text { i1 }+=\text { incl; } \\
& \text { i2 }+=\text { inc2; } \\
& \text { i } 3+=\text { inc } 3 \text {; } \\
& \text { \} }
\end{aligned}
$$

From the reasoning above, it might be thought that this code would not vectorize, since the values of i1, i2, and i3 are computed iteratively, like the values of the pointers in the first example. The compiler, however, happily vectorizes this version, evidently treating pointer variables differently from indices! The confusing nature of this example illustrates the degree to which programming supercomputers is still something of a black art. Because of this, it is desirable to use subroutines from standard mathematical libraries (that have already been optimized and tested by someone else) whenever possible.

In summary, the large capacity of supercomputers makes them possibly the only choice for simulating very large systems. For medium-sized problems, faster execution speed may not be adequate compensation for the extra programming effort required. The use of high-level interpretive systems is recommended both to make applications portable and to minimize the amount of compiled code that must be maintained. revision accepted for publication January 22,1996 .) 